\title{
Nuclear electromagnetic charge and current operators in Chiral EFT
}

\author{
Luca Girlanda* \\ Dipartimento di Matematica e Fisica "E. De Giorgi", Università del Salento, \\ INFN Sez. di Lecce, 73100 Italy \\ E-mail: luca.girlanda@le.infn.it
}

\section{Laura Elisa Marcucci}

Dipartimento di Fisica "E. Fermi", Università di Pisa,

INFN Sez. di Pisa, 56127 Italy

E-mail: marcucci@df.unipi.it

\section{Saori Pastore}

Department of Physics and Astronomy, University of South Carolina, Columbia, SC 29208 USA

E-mail: pastoresemailbox.sc.edu

\section{Maria Piarulli}

Department of Physics, Old Dominion University, Norfolk, VA 23529 USA

E-mail: mpiar001@odu.edu

\section{Rocco Schiavilla}

Theory Center, Jefferson Laboratory, Newport News, VA 23606 USA

Department of Physics, Old Dominion University, Norfolk, VA 23529 USA

E-mail: schiavilajlab.org

\section{Michele Viviani}

INFN Sez. di Pisa, 56127 Italy

E-mail: viviani@pi.infn.it

\begin{abstract}
We describe our method for deriving the nuclear electromagnetic charge and current operators in chiral perturbation theory, based on time-ordered perturbation theory. We then discuss possible strategies for fixing the relevant low-energy constants, from the magnetic moments of the deuteron and of the trinucleons, and from the radiative $n p$ capture cross sections, and identify a scheme which, partly relying on $\Delta$ resonance saturation, leads to a reasonable pattern of convergence of the chiral expansion.
\end{abstract}

The 7th International Workshop on Chiral Dynamics

August 6 -10, 2012

Jefferson Lab, Newport News, Virginia, USA

${ }^{*}$ Speaker. 


\section{Introduction}

One of the great advantages of the chiral effective field theory $(\chi$ EFT) description of the nuclear interaction at low energy is the possibility to derive electroweak currents consistently with the interactions. Indeed, the chiral symmetry of strong interactions severely constrains the form of both, since electroweak probes are coupled to its Noether currents. Within the effective theory, chiral symmetry constraints are implemented requiring the Lagrangian to be invariant under local chiral transformations [1], with the external currents $r_{\mu}, \ell_{\mu}$ transforming as the corresponding gauge fields. Explicit chiral symmetry breaking can be taken into account by setting the scalar source $\chi$ proportional to the quark mass matrix, while the coupling to the external photons $A_{\mu}$ is obtained through $r_{\mu}=\ell_{\mu}=Q A_{\mu}$ where $Q=e \operatorname{diag}\left(\frac{2}{3},-\frac{1}{3}\right)$ in the meson sector and $Q=e \operatorname{diag}(1,0)$ in the nucleon sector. The effective Lagrangian is then ordered in a combined expansion (chiral expansion) in powers of quark masses and small momenta, where $\chi \sim O\left(p^{2}\right)$.

The Hamiltonians describing the interactions of pions, nucleons, and photons, are derived from the chiral Lagrangians [2] in the canonical formalism. The relevant contributions for our discussion read

$$
\begin{aligned}
H_{\pi N}= & \int \mathrm{d} \mathbf{x} N^{\dagger}\left[\frac{g_{A} \tau_{a}}{F_{\pi}} \boldsymbol{\sigma} \cdot \nabla \pi_{a}+\frac{\boldsymbol{\tau}}{F_{\pi}^{2}} \cdot\left(\boldsymbol{\pi} \times \partial^{0} \boldsymbol{\pi}\right)+\ldots\right] N, \\
H_{\gamma N}= & e \int \mathrm{d} \mathbf{x} N^{\dagger}\left[e_{N} A^{0}+i \frac{e_{N}}{2 m}(-\overleftarrow{\nabla} \cdot \mathbf{A}+\mathbf{A} \cdot \vec{\nabla})-\frac{\mu_{N}}{2 m} \boldsymbol{\sigma} \cdot \boldsymbol{\nabla} \times \mathbf{A}\right. \\
& \left.-\frac{2 \mu_{N}-e_{N}}{8 m^{2}}\left(\nabla^{2} A^{0}+\boldsymbol{\sigma} \times \boldsymbol{\nabla} A^{0} \cdot \vec{\nabla}-\overleftarrow{\nabla} \cdot \boldsymbol{\sigma} \times \boldsymbol{\nabla} A^{0}\right)+\ldots\right] N \\
H_{\gamma \pi}= & e \int \mathrm{d} \mathbf{x}\left[A^{0}\left(\boldsymbol{\pi} \times \partial^{0} \boldsymbol{\pi}\right)_{z}+\varepsilon_{z a b} \pi_{a}\left(\boldsymbol{\nabla} \pi_{b}\right) \cdot \mathbf{A}+\ldots\right] \\
H_{\gamma \pi N}= & e \int \mathrm{d} \mathbf{x} N^{\dagger}\left[-\frac{g_{A}}{F_{\pi}}(\boldsymbol{\tau} \times \boldsymbol{\pi})_{z} \boldsymbol{\sigma} \cdot \mathbf{A}+\frac{g_{A}}{2 m F_{\pi}}\left(\boldsymbol{\tau} \cdot \boldsymbol{\pi}+\pi_{z}\right) \boldsymbol{\sigma} \cdot \boldsymbol{\nabla} A^{0}\right. \\
& \left.+\left(\frac{d_{8}^{\prime}}{F_{\pi}} \nabla \pi_{z}+\frac{d_{9}^{\prime}}{F_{\pi}} \tau_{a} \nabla \pi_{a}+\frac{d_{21}^{\prime}}{F_{\pi}} \varepsilon_{z a b} \tau_{b} \boldsymbol{\sigma} \times \boldsymbol{\nabla} \pi_{a}\right) \cdot \boldsymbol{\nabla} \times \boldsymbol{A}+\ldots\right] N
\end{aligned}
$$

where the parameters $d_{i}$ are (unknown) low-energy constants (LECs), the isospin operators $e_{N}$ and $\mu_{N}$ are defined as

$$
e_{N}=\left(1+\tau_{z}\right) / 2, \kappa_{N}=\left(\kappa_{S}+\kappa_{V} \tau_{z}\right) / 2, \mu_{N}=e_{N}+\kappa_{N},
$$

and $\kappa_{S}$ and $\kappa_{V}$ are the isoscalar and isovector combinations of the anomalous magnetic moments of the proton and neutron. The isospin doublet of (non-relativistic) nucleon fields, isospin triplet of pion fields, and electromagnetic (EM) vector field are denoted by $N, \boldsymbol{\pi}$, and $A^{\mu}$, respectively, and $\boldsymbol{\sigma}$ and $\tau$ are spin and isospin Pauli matrices. The arrow over the gradient specifies whether it acts on the left or right nucleon field. Only terms relevant for the construction of the two-nucleon potential and/or EM charge and current operators at one loop are retained in the interaction Hamiltonians listed above. The naive power counting of the resulting vertices follows by noting that each gradient brings in a factor of $Q$, where $Q$ is the low-momentum scale, so, for example, the two terms in $H_{\pi N}$ are each of order $\sim Q$, while the first terms in $H_{\gamma \pi N}$ are of order $\sim e Q^{0}$ and $\sim e Q$, and the remaining ones in second line of Eq. (1.4) are of order $\sim e Q^{2}$. 
Two-nucleon contact Hamiltonians can conveniently be written in terms of the standard set of operators introduced in Ref. [3] as

$$
\begin{aligned}
H_{C T}= & \int \mathrm{d} \mathbf{x}\left[\frac{1}{2} C_{S} O_{S}+\frac{1}{2} C_{T} O_{T}+\frac{1}{2} C_{1}\left(O_{1}+2 O_{2}\right)-\frac{1}{8} C_{2}\left(2 O_{2}+O_{3}\right)\right. \\
& +\frac{1}{2} C_{3}\left(O_{9}+2 O_{12}\right)+\frac{1}{8} C_{4}\left(O_{9}+O_{14}\right)-\frac{1}{4} C_{5}\left(O_{6}-O_{5}\right) \\
& \left.+\frac{1}{2} C_{6}\left(O_{7}+2 O_{10}\right)+\frac{1}{16} C_{7}\left(O_{7}+O_{8}+2 O_{13}\right)\right]
\end{aligned}
$$

where the $C_{i}$ are LECs which are typically determined by fitting low-energy two-nucleon scattering data and the deuteron binding energy, and relativistic corrections have been ignored. Of course, minimal substitution $\boldsymbol{\nabla} N \rightarrow\left(\boldsymbol{\nabla}-i e e_{N} \mathbf{A}\right) N$ in $H_{C T}$ leads to a (contact) Hamiltonian $H_{C T}^{\gamma \mathrm{m}}$ which includes the coupling to the EM field and implies a (two-nucleon) contact current operator. However, non-minimal couplings through the electromagnetic field tensor $F_{\mu \nu}$ are also allowed. It can be shown that the only two independent operator structures are

$$
H_{C T}^{\gamma \mathrm{nm}}=e \int \mathrm{d} \mathbf{x}\left[C_{15}^{\prime} N^{\dagger} \boldsymbol{\sigma} N N^{\dagger} N+C_{16}^{\prime}\left(N^{\dagger} \boldsymbol{\sigma} \tau_{z} N N^{\dagger} N-N^{\dagger} \boldsymbol{\sigma} N N^{\dagger} \tau_{z} N\right)\right] \cdot \boldsymbol{\nabla} \times \mathbf{A},
$$

where the isoscalar $C_{15}^{\prime}$ and isovector $C_{16}^{\prime}$ LECs (as well as the $d_{i}$ 's multiplying the higher order terms in the $\gamma \pi N$ Hamiltonian) can be determined by fitting photo-nuclear data in the few-nucleon systems.

\section{From amplitudes to potentials}

The transition amplitudes obtained from the above Hamiltonians can be expanded in terms of $Q / \Lambda_{\chi}$, where $Q \ll \Lambda_{\chi}$ is the pion momentum-coupling and $\Lambda_{\chi} \sim 1 \mathrm{GeV}$ is the chiral-symmetry breaking scale. For each given order of the expansion, the number of terms contributing to the amplitude is finite [4]. Nuclear force and current operators, are then extracted from the transition amplitude, which can be represented by time-ordered diagrams scaling as a power of $Q / \Lambda_{\chi}$.

Reducible diagrams, i.e. diagrams which involve purely nucleonic intermediate state, are enhanced compared to irreducible diagrams by a factor of $Q$ for each purely nucleonic intermediate state. This feature spoils the power counting scheme. In addition, within the static limit-that is in the limit in which $m \rightarrow \infty$, or equivalently neglecting nucleon kinetic energies-reducible contributions are infrared-divergent. According to the prescription proposed by Weinberg[4] nuclear force (and current) operators are given by the irreducible contributions only. Reducible contributions, instead, are generated by solving the Schrödinger equation iteratively with the nuclear potential (and current) operator arising from the irreducible amplitude.

Our formalism follows this prescription. However, the omission of reducible contributions from the definition of nuclear operators needs to be dealt with care when the irreducible amplitude is evaluated under an approximation. For example, if the irreducible amplitude is evaluated under the static limit approximation (which is usually the case) then the iterative process will generate only part of the reducible amplitude (i.e. the one which includes the approximate static nuclear operators). The reducible part of the amplitude which is not generated by iteration, in this case 
the one that is obtained going beyond the static limit approximation, needs to be incorporated order by order-along with the irreducible amplitude - in the definition of nuclear operators. This scheme, in combination with time-ordered perturbation theory (TOPT)—which is best suited to separate the reducible content from the irreducible one, has been implemented in Refs. [5, 6, 7]. This method leads to nuclear operators which are not-uniquely defined due to the not-uniqueness of the transition amplitude off-the-energy shell. However the operators, although not-unique, are unitarily equivalent, therefore the description of physical systems is not affected by this ambiguity.

Another approach, implemented to face the difficulties posed by the reducible amplitude behavior, has been introduced by Okubo [8] and extensively developed by Epelbaum and collaborators [9]. The method, referred to as the unitary transformation method, is based on TOPT and exploits a unitary transformation to decouple the Fock space of pions and nucleons into two subspaces, one containing only pure-nucleonic states and the other one involving states which contemplate at least one pion. In this decoupled space, the amplitude does not involve enhanced contributions associated with the reducible diagrams. The subspaces are not-uniquely defined because it is always possible to perform additional unitary transformations onto them, with a consequent change in the formal definition of the resulting nuclear operators. This, of course, does not affect the physical representations. Nuclear electromagnetic charge and current operators within this scheme have been developed in Refs. [10,11], including the complete renormalization of the tree-level currents. This makes it possible to carry fully consistent ChEFT calculations of nuclear electromagnetic observables, using nuclear wave functions arising from the potential developed within the same scheme. It is worthwhile to mention that the first set of EM nuclear current operators derived within a pion-nucleon $\chi$ EFT framework has been provided by Park, Min, and Rho in Ref. [12]. In that work, the authors carry out the calculation of the transition amplitude in covariant perturbation theory. The similarities and differences between this last approach and those based on TOPT described above have been discussed in detail in Refs. [5, 6].

In what follows, we focus on the method developed in Refs. [5, 6, 7] and show how nuclear operators are extracted from the transition amplitude. We are especially interested in the construction of the EM transition operators, but due to the aforementioned ambiguity in the definition of the off-the-energy shell amplitude, we need to derive the nuclear potential along with the EM transition operators. This allows us to correctly separate the iterative content of the reducible amplitude from the genuine one contributing to the definition of the operators.

The conventional perturbative expansion for the two-nucleon $(N N)$ scattering amplitude reads

$$
\langle f|T| i\rangle=\left\langle f\left|H_{1} \sum_{n=1}^{\infty}\left(\frac{1}{E_{i}-H_{0}+i \eta} H_{1}\right)^{n-1}\right| i\right\rangle,
$$

where $|i\rangle$ and $|f\rangle$ represent the initial and final $N N$ states of energy $E_{i}=E_{f}, H_{0}$ is the Hamiltonian describing free pions and nucleons, and $H_{1}$ is the Hamiltonian describing interactions among these particles (Sec. 1). The evaluation of this amplitude is carried out in practice by inserting complete sets of $H_{0}$ eigenstates between successive terms of $H_{1}$. Power counting is then used to organize the expansion in powers of $Q / \Lambda_{\chi} \ll 1$, where $\Lambda_{\chi} \simeq 1 \mathrm{GeV}$ is the typical hadronic mass scale.

In the perturbative series, Eq. (2.1), a generic (reducible or irreducible) contribution is characterized by a certain number, say $N$, of vertices, each scaling as $Q^{\alpha_{i}} \times Q^{-\beta_{i} / 2}(i=1, \ldots, N)$, where $\alpha_{i}$ is the power counting implied by the relevant interaction Hamiltonian and $\beta_{i}$ is the number of 
pions in and/or out of the vertex, a corresponding $N-1$ number of energy denominators, and possibly $L$ loops. Out of these $N-1$ energy denominators, $N_{K}$ of them will involve only nucleon kinetic energies, which scale as $Q^{2}$, and the remaining $N-N_{K}-1$ will involve, in addition, pion energies, which are of order $Q$. Loops, on the other hand, contribute a factor $Q^{3}$ each, since they imply integrations over intermediate three momenta. Hence the power counting associated with such a contribution is

$$
\left(\prod_{i=1}^{N} Q^{\alpha_{i}-\beta_{i} / 2}\right) \times\left[Q^{-\left(N-N_{K}-1\right)} Q^{-2 N_{K}}\right] \times Q^{3 L} .
$$

Clearly, each of the $N-N_{K}-1$ energy denominators can be further expanded as

$$
\frac{1}{E_{i}-E_{I}-\omega_{\pi}}=-\frac{1}{\omega_{\pi}}\left[1+\frac{E_{i}-E_{I}}{\omega_{\pi}}+\frac{\left(E_{i}-E_{I}\right)^{2}}{\omega_{\pi}^{2}}+\ldots\right],
$$

where $E_{I}$ denotes the kinetic energy of the intermediate two-nucleon state, $\omega_{\pi}$ the pion energy (or energies, as the case may be), and the ratio $\left(E_{i}-E_{I}\right) / \omega_{\pi}$ is of order $Q$.

The $Q$-scaling of the interaction vertices and the considerations above show that $T$ admits the following expansion:

$$
T=T^{(0)}+T^{(1)}+T^{(2)}+\ldots,
$$

where $T^{(n)} \sim Q^{n}$. A two-nucleon potential $v$ can then be derived which, when iterated in the Lippmann-Schwinger (LS) equation,

$$
v+v G_{0} v+v G_{0} v G_{0} v+\ldots,
$$

leads to the on-the-energy-shell $\left(E_{i}=E_{f}\right) T$-matrix in Eq. (2.4), order by order in the power counting. In Eq. (2.5), $G_{0}$ denotes the free two-nucleon propagator, $G_{0}=1 /\left(E_{i}-E_{I}+i \eta\right)$, and we assume that

$$
v=v^{(0)}+v^{(1)}+v^{(2)}+\ldots,
$$

where the yet to be determined $v^{(n)}$ is of order $Q^{n}$. We also note that, generally, a term like $v^{(m)} G_{0} v^{(n)}$ is of order $Q^{m+n+1}$, since $G_{0}$ is of order $Q^{-2}$ and the implicit loop integration brings in a factor $Q^{3}$. Having established the above power counting, we obtain

$$
\begin{aligned}
v^{(0)} & =T^{(0)} \\
v^{(1)}=T^{(1)}- & {\left[v^{(0)} G_{0} v^{(0)}\right] } \\
v^{(2)}= & T^{(2)}-\left[v^{(0)} G_{0} v^{(0)} G_{0} v^{(0)}\right]-\left[v^{(1)} G_{0} v^{(0)}+v^{(0)} G_{0} v^{(1)}\right] \\
v^{(3)}=T^{(3)}- & {\left[v^{(0)} G_{0} v^{(0)} G_{0} v^{(0)} G_{0} v^{(0)}\right]-\left[v^{(1)} G_{0} v^{(0)} G_{0} v^{(0)}+\text { permutations }\right] } \\
& -\left[v^{(2)} G_{0} v^{(0)}+v^{(0)} G_{0} v^{(2)}\right]-\left[v^{(1)} G_{0} v^{(1)}\right] .
\end{aligned}
$$

Since the potential is thus defined from on-shell amplitudes order by order, different off-shell extensions of the potential at a given order lead to different (on-shell) potentials at higher orders. In fact, there is an infinite class of $v^{(2)}(v)$ non-static corrections-labeled by the parameter $v[13,14,15,7]-$ which, while equivalent on the energy-shell, are different off the energy-shell, and therefore lead to different potentials $v^{(3)}(v)$ in Eq. (2.10). However, this ambiguity is of no consequence, since 
it can be shown that different off-the-energy-shell extrapolations $v^{(2)}(v)$ and $v^{(3)}(v)$ are unitarily equivalent $[13,7]$.

The inclusion (in first order) of electromagnetic interactions in the perturbative expansion of Eq. (2.1) is in principle straightforward. The transition operator can be expanded as

$$
T_{\gamma}=T_{\gamma}^{(-3)}+T_{\gamma}^{(-2)}+T_{\gamma}^{(-1)}+\ldots
$$

where $T_{\gamma}^{(n)}$ is of order $e Q^{n}$ ( $e$ is the electric charge). The nuclear charge, $\rho$, and current, $\mathbf{j}$, operators follow from $v_{\gamma}=A^{0} \rho-\mathbf{A} \cdot \mathbf{j}$, where $A^{\mu}=\left(A^{0}, \mathbf{A}\right)$ is the electromagnetic vector field, and it is assumed that $v_{\gamma}$ has a similar expansion as $T_{\gamma}$. The requirement that, in the context of the LS equation, $v_{\gamma}$ matches $T_{\gamma}$ order by order in the power counting implies the following relations:

$$
\begin{aligned}
v_{\gamma}^{(-3)}=T_{\gamma}^{(-3)} & \\
v_{\gamma}^{(-2)}=T_{\gamma}^{(-2)}- & {\left[v_{\gamma}^{(-3)} G_{0} v^{(0)}+v^{(0)} G_{0} v_{\gamma}^{(-3)}\right] } \\
v_{\gamma}^{(-1)}=T_{\gamma}^{(-1)}- & {\left[v_{\gamma}^{(-3)} G_{0} v^{(0)} G_{0} v^{(0)}+\text { permutations }\right]-\left[v_{\gamma}^{(-2)} G_{0} v^{(0)}+v^{(0)} G_{0} v_{\gamma}^{(-2)}\right], } \\
v_{\gamma}^{(0)}=T_{\gamma}^{(0)}- & {\left[v_{\gamma}^{(-3)} G_{0} v^{(0)} G_{0} v^{(0)} G_{0} v^{(0)}+\text { permutations }\right] } \\
- & {\left[v_{\gamma}^{(-2)} G_{0} v^{(0)} G_{0} v^{(0)}+\text { permutations }\right] } \\
- & {\left[v_{\gamma}^{(-1)} G_{0} v^{(0)}+v^{(0)} G_{0} v_{\gamma}^{(-1)}\right]-\left[v_{\gamma}^{(-3)} G_{0} v^{(2)}+v^{(2)} G_{0} v_{\gamma}^{(-3)}\right] } \\
v_{\gamma}^{(1)}=T_{\gamma}^{(1)}- & {\left[v_{\gamma}^{(-3)} G_{0} v^{(0)} G_{0} v^{(0)} G_{0} v^{(0)} G_{0} v^{(0)}+\text { permutations }\right] } \\
- & {\left[v_{\gamma}^{(-2)} G_{0} v^{(0)} G_{0} v^{(0)} G_{0} v^{(0)}+\text { permutations }\right] } \\
- & {\left[v_{\gamma}^{(-1)} G_{0} v^{(0)} G_{0} v^{(0)}+\text { permutations }\right]-\left[v_{\gamma}^{(0)} G_{0} v^{(0)}+v^{(0)} G_{0} v_{\gamma}^{(0)}\right] } \\
- & {\left[v_{\gamma}^{(-3)} G_{0} v^{(2)} G_{0} v^{(0)}+\text { permutations }\right]-\left[v_{\gamma}^{(-3)} G_{0} v^{(3)}+v^{(3)} G_{0} v_{\gamma}^{(-3)}\right] }
\end{aligned}
$$

where $v_{\gamma}^{(n)}=A^{0} \rho^{(n)}-\mathbf{A} \cdot \mathbf{j}^{(n)}, v^{(n)}$ are the $N N$ potentials constructed in Eqs. (2.7)-(2.10) (the $v$ dependence of $v^{(2)}$ and $v^{(3)}$ is understood), and use has been made of the fact that $v^{(1)}$ vanishes. In the propagator $G_{0}$, the initial energy $E_{i}$ includes the photon energy $\omega_{\gamma}$ (itself of order $Q^{2}$ ), since $E_{i}=E_{1}+E_{2}+\omega_{\gamma}=E_{1}^{\prime}+E_{2}^{\prime}$, and the intermediate energy $E_{I}$ may include, in addition to the kinetic energies of the intermediate nucleons, also the photon energy, depending on the specific time ordering being considered.

\section{Electromagnetic current and charge operators up to one loop}

The contributions to the electromagnetic current operator up to one loop are illustrated diagrammatically in Fig. 1. They follow from the interaction Hamiltonians listed in Sec. 1 and the perturbative expansion for $v_{\gamma}^{(n)}$ in Eqs. (2.13)-(2.16). There is no $n=-3$ contribution to $\mathbf{j}$, therefore the current operator is unaffected by the non-static corrections entering the potentials $v^{(2)}$ and $v^{(3)}$. The lowest order $(n=-2)$ consists of the single-nucleon convection and spin-magnetization currents:

$$
\mathbf{j}^{(-2)}=\frac{e}{2 m}\left(2 e_{N, 1} \mathbf{K}_{1}+i \mu_{N, 1} \boldsymbol{\sigma}_{1} \times \mathbf{q}+1 \rightleftharpoons 2\right),
$$


where $\mathbf{q}$ is the momentum carried by the external field, $\mathbf{k}_{i}$ and $\mathbf{K}_{i}$ denote the combinations of initial and final nucleon momenta $\mathbf{k}_{i}=\mathbf{p}_{i}^{\prime}-\mathbf{p}_{i}, \mathbf{K}_{i}=\left(\mathbf{p}_{i}^{\prime}+\mathbf{p}_{i}\right) / 2$, and $e_{N, i}$ and $\mu_{N, i}$ have been defined in Eq. (1.5). The counting $e Q^{-2}$ follows from the product of a factor $e Q^{1}$ associated with the $\gamma N N$ vertex, and a factor $Q^{-3}$ due to the momentum-conserving $\delta$-function implicit in a disconnected term of this type. We refer to Refs. $[5,6,16]$ for the explicit expressions up to N3LO, as obtained

$$
\begin{aligned}
& { }^{e Q^{-2}} \mid \mathrm{Z} \\
& e^{-1} \quad z^{-} \mid z^{-} \\
& e Q^{\circ}
\end{aligned}
$$

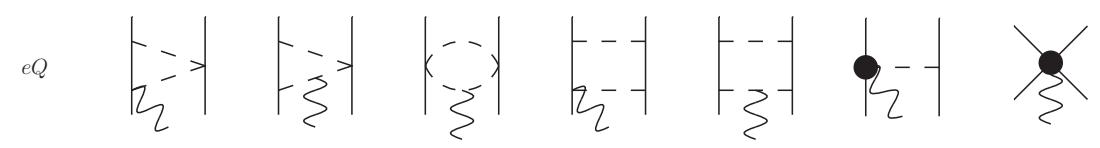

Figure 1: Diagrams illustrating one- and two-body currents entering at LO $\left(e Q^{-2}\right), \mathrm{NLO}\left(e Q^{-1}\right), \mathrm{N} 2 \mathrm{LO}$ $\left(e Q^{0}\right)$, and N3LO $\left(e Q^{1}\right)$. Nucleons, pions, and photons are denoted by solid, dashed, and wavy lines. Only the relevant topologies are indicated. Loop corrections to short-range currents turn out to vanish.

within the formalism outlined in Sec. 2. They depend on the known parameters $g_{A}$ and $F_{\pi}$ (NLO and N3LO), and the nucleon's magnetic moments (LO and N2LO). Loop corrections to the shortrange currents turn out to cancel [16]. Unknown LECs enter the N3LO OPE contribution involving the $\gamma \pi N$ vertex of order $e Q^{2}$ from $H_{\gamma \pi N}$,

$$
\mathbf{j}_{\gamma \pi N}^{(1)}=i e \frac{g_{A}}{F_{\pi}^{2}} \frac{\boldsymbol{\sigma}_{2} \cdot \mathbf{k}_{2}}{\omega_{k_{2}}^{2}}\left[\left(d_{8}^{\prime} \tau_{2, z}+d_{9}^{\prime} \boldsymbol{\tau}_{1} \cdot \boldsymbol{\tau}_{2}\right) \mathbf{k}_{2}-d_{21}^{\prime}\left(\boldsymbol{\tau}_{1} \times \boldsymbol{\tau}_{2}\right)_{z} \boldsymbol{\sigma}_{1} \times \mathbf{k}_{2}\right] \times \mathbf{q}+1 \rightleftharpoons 2 .
$$

The contributing LECs could be fixed by relating them, in a resonance saturation picture, to the couplings in the $N$ to $\Delta$ excitation and $\rho \pi \gamma$ transition currents, or they could be fixed by pion photo-production data on a single nucleon or photo-nuclear data at low energies. Further LECs enter the two-contact currents, from minimal and non-minimal substitution, using Fierz identities they can be written as

$$
\begin{aligned}
\mathbf{j}_{\mathrm{CT}, \mathrm{m}}^{(1)}= & \frac{i}{16}\left(\boldsymbol{\tau}_{1} \times \boldsymbol{\tau}_{2}\right)_{z}\left[\left[C_{2}+3 C_{4}+C_{7}+\left(C_{2}-C_{4}-C_{7}\right) \boldsymbol{\sigma}_{1} \cdot \boldsymbol{\sigma}_{2}\right]\left(\mathbf{k}_{1}-\mathbf{k}_{2}\right)\right. \\
& \left.+C_{7}\left[\boldsymbol{\sigma}_{1} \cdot\left(\mathbf{k}_{1}-\mathbf{k}_{2}\right) \boldsymbol{\sigma}_{2}+\boldsymbol{\sigma}_{2} \cdot\left(\mathbf{k}_{1}-\mathbf{k}_{2}\right) \boldsymbol{\sigma}_{1}\right]\right] \\
& -\frac{i C_{5}}{4}\left(\boldsymbol{\sigma}_{1}+\boldsymbol{\sigma}_{2}\right) \times\left(e_{1} \mathbf{k}_{1}+e_{2} \mathbf{k}_{2}\right) .
\end{aligned}
$$

and

$$
\mathbf{j}_{\mathrm{CT}, \mathrm{nm}}^{(1)}=-i e\left[C_{15}^{\prime} \boldsymbol{\sigma}_{1}+C_{16}^{\prime}\left(\tau_{1, z}-\tau_{2, z}\right) \boldsymbol{\sigma}_{1}\right] \times \mathbf{q}+1 \rightleftharpoons 2
$$


No three-body currents arise up to $\mathrm{N}^{3} \mathrm{LO}$ included here, due to cancellations between irreducible and recoil-corrected reducible diagrams, similarly to what happens for the three-nucleon force.

Contributions to the two-body charge operators $\rho^{(n)}$ up to order $e Q^{1}$ (N4LO) included are illustrated in Fig. 2. The $e Q^{-3}$ (LO) operator, resulting from the first term of the $\gamma N$ interaction Hamiltonian in Eq. (1.2), is

$$
\rho^{(-3)}=e e_{N, 1}+1 \rightleftharpoons 2 .
$$

There are no NLO $\left(e Q^{-2}\right)$ contributions, whereas at N2LO there is a relativistic correction of or$\operatorname{der}(Q / m)^{2}$ to the LO charge operator, which results from the second line of Eq. (1.2). At this order, there are in principle also a pion-in-flight term, which, however, turns out to vanish when the contributions of the six time-ordered diagrams, evaluated in the static limit, are summed up, and a one-pion-exchange (OPE) contribution, which vanishes due to a similar cancellation. We

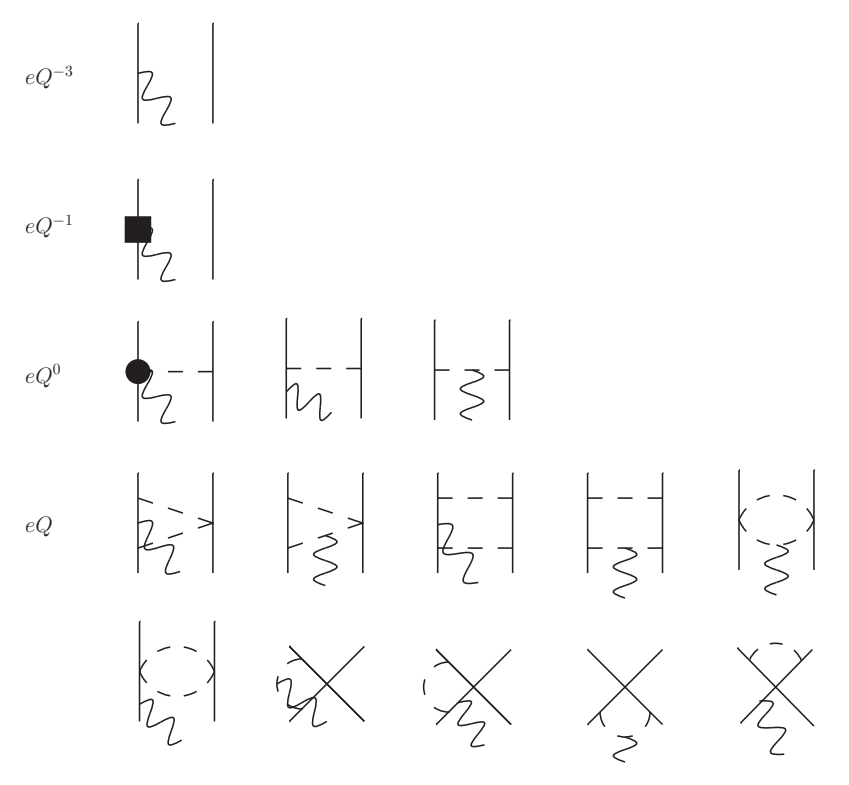

Figure 2: Diagrams illustrating one- and two-body charge operators entering at $\mathrm{LO}\left(e Q^{-3}\right), \mathrm{N} 2 \mathrm{LO}\left(e Q^{-1}\right)$, $\mathrm{N} 3 \mathrm{LO}\left(e Q^{0}\right)$, and N4LO $\left(e Q^{1}\right)$. The square represents the $(Q / m)^{2}$, or $(v / c)^{2}$, relativistic correction to the LO one-body charge operator, whereas the solid circle is associated with a $\gamma \pi N$ charge coupling of order $e Q$. Only the relevant topologies are indicated.

note that the power counting is different for the current operator, for which the LO term is of order $e Q^{-2}$ (in the two-nucleon system), i.e. it is suppressed by an extra power of $Q$ relative to $\rho^{(-3)}$, and where there are $\mathrm{NLO}\left(e Q^{-1}\right)$ corrections involving seagull and in-flight contributions associated with OPE, which have no counterpart in the present case. The pion-in-flight and OPE diagrams also lead to N3LO contributions due to non-static corrections resulting from the expansion of the denominators involving pion energies as in Eq. (2.3). In particular, the specific form of the N3LO charge operator depends on the (non-unique) off-the-energy shell prescription adopted for the non-static piece in the OPE potential [7]. The same applies to part of the N4LO contributions. This ambiguity in the non-static OPE and TPE potentials and accompanying charge operators is of no consequence, however, since different form for these are related to each other by a unitary 
transformation[7, 16]. Thus, provided a consistent set is adopted, predictions for physical observables, such as the few-nucleon charge form factors, will remain unaffected by the non-uniqueness associated with off-the-energy-shell effects. We note that, while the loop integrals entering (the non-vanishing) diagrams at N4LO are individually ultra-violet divergent, their sum is finite, i.e. the divergencies cancel out. This is in line with the fact that there are no counter-terms at this order. Finally, charge conservation,

$$
\rho(\mathbf{q}=0)=\int \mathrm{d} \mathbf{x} \rho(\mathbf{x})=e\left(e_{1}+e_{2}\right)
$$

where $\rho(\mathbf{x}$ and $\rho(\mathbf{q})$ denote, respectively, the charge density and its Fourier transform, implies that the charge operators $\rho^{(n \geq-2)}(\mathbf{q})$ vanish at $\mathbf{q}=0$. This latter requirement is satisfied by the operators illustrated in Fig. 2, regardless of the adopted off-the-energy shell prescription[7]. We also emphasize that, up to N4LO included, there are no unknown LECs.

\section{Fixing the LECs}

The operators described in the previous section depend on the LECs $C_{i}$ entering the twonucleon contact Lagrangian through the minimal coupling procedure, on the non-minimal coupling LECs $C_{15}^{\prime}$ and $C_{16}^{\prime}$ and on the subleading pion-nucleon couplings $d_{i}^{\prime}$ entering in the one-pion exchange N3LO current. The latter could be fitted to pion photo-production data on a single nucleon, which however involve photon energies much higher than those relevant for the threshold processes under consideration here, or related to hadronic coupling constants through resonance saturation. We prefer to treat them as fitting parameters, assuming however $d_{21}^{\prime}=d_{8}^{\prime} / 4$ as suggested by $\Delta$ resonance dominance. We are then left with 4 parameters, two isoscalar and two isovector, that we express as adimensional LECs $d_{i}^{S / V}$,

$$
\begin{array}{ll}
C_{15}^{\prime}=d_{1}^{S} / \Lambda^{4}, & d_{9}^{\prime}=d_{2}^{S} / \Lambda^{2}, \\
C_{16}^{\prime}=d_{1}^{V} / \Lambda^{4}, & d_{8}^{\prime}=d_{2}^{V} / \Lambda^{2},
\end{array}
$$

In Ref.[17] we fixed $d_{i}^{S}$ to the deuteron and isoscalar trinucleon magnetic moment, and $d_{i}^{V}$ to the $n p$ thermal radiative capture cross section and isovector trinucleon magnetic moment, using accurate nuclear wave functions obtained from the Hyperspherical Harmonic method [18]. We used the Argonne $v_{18}$ (AV18) [19] and the Idaho chiral N3LO [20] two-nucleon potential, supplemented with the Urbana-IX [21] and chiral N2LO [22] three-nucleon interaction. We were then able to obtain prediction in good agreement with experimental data for the thermal $n d$ and $n-{ }^{3} \mathrm{He}$ radiative capture cross sections $\left(\sigma_{n d}^{\gamma}\right.$ and $\sigma_{n-3}^{\gamma} \mathrm{He}$ ) and the photon circular polarization parameter $R_{c}$ in the capture of polarized neutrons on deuterons (we should point out that the adopted model for the current included loop corrections to the short range contributions which instead turn out to be absent [16]). Here we make use of the more recent N3LO chiral two-nucleon potential[23], available for two values of the cutoff $\Lambda=500,600 \mathrm{MeV}$, taking from this reference the LECs $C_{i}$ entering the current operators through the minimal coupling procedure. As before, the isoscalar LECs $d_{i}^{S}$ are fixed from the deuteron and the isoscalar combination of the trinucleon magnetic moments, resulting in the values displayed in Table 1. Eventhough the LEC of the contact term turns out 


\begin{tabular}{ccc}
\hline$\Lambda$ & $d_{1}^{S}$ & $10 \cdot d_{2}^{S}$ \\
500 & $4.072(2.522)$ & $2.190(-1.731)$ \\
600 & $11.38(5.238)$ & $3.231(-2.033)$ \\
\hline
\end{tabular}

Table 1: Extracted values of the isoscalar LECs from the deuteron and trinucleon isoscalar magnetic moments for different values of the cutoff $\Lambda$, using the N3LO/N2LO chiral two/three nucleon potential (AV18+UIX in parentheses) to generate the nuclear wave functions.

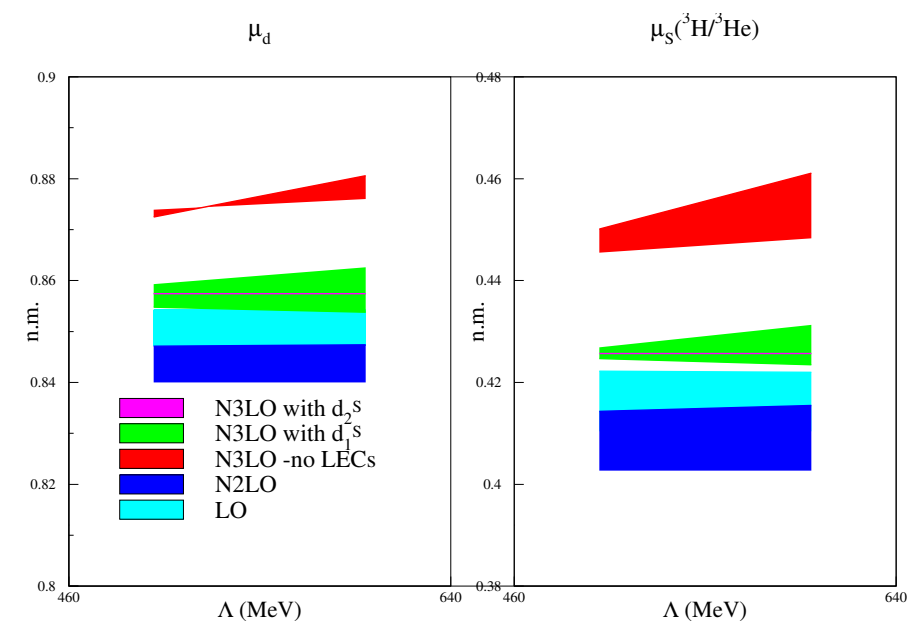

Figure 3: Cumulative contributions to the deuteron and trinucleon (isoscalar) magnetic moments. The bands represent the variation with the two nuclear Hamiltonian model.

\begin{tabular}{c|cc|cc|cc}
\hline$\Lambda$ & $d_{1}^{V}(I)$ & $d_{2}^{V}(I)$ & $d_{1}^{V}(I I)$ & $d_{2}^{V}(I I)$ & $d_{1}^{V}(I I I)$ & $d_{2}^{V}(I I I)$ \\
500 & $10.36(45.10)$ & $17.42(35.57)$ & $-13.30(-9.339)$ & 3.458 & $-7.981(-5.187)$ & 3.458 \\
600 & $41.84(257.5)$ & $33.14(75.00)$ & $-22.31(-11.57)$ & 4.980 & $-11.69(-1.025)$ & 4.980 \\
\hline
\end{tabular}

Table 2: Extracted values for the isovector LECs as explained in the text. Notation as in Table 1.

to be rather large, the convergence pattern for these observables is reasonable, as shown in Fig. 3 (there are no contributions from N2LO and from the loops, which are isovector).

On the contrary, an analogous strategy to fix the isovector LECs from the $n p$ radiative capture cross section and isovector trinucleon magnetic moment results in unreasonably large values for $d_{1,2}^{V}$ (denoted as set I, first column of Table 4), which totally spoil the convergence pattern. Instead, in sets II and III, the LEC $d_{2}^{V}$ is fixed by assuming $\Delta$-resonance dominance,

$$
d_{2}^{V}=\frac{4 \mu^{*} h_{A} \Lambda^{2}}{9 m\left(m_{\Delta}-m\right)}
$$

where $m_{\Delta}-m=294 \mathrm{MeV}, h_{A} / F_{\pi}=f_{\pi N \Delta} / m_{\pi}$ with $f_{\pi N \Delta}^{2} /(4 \pi)=0.35$ from the $\Delta$ width, and the transition magnetic moment $\mu^{*}=3 \mathrm{n} . \mathrm{m}$. from an analysis of pion photo-production data in the $\Delta$ region. On the other hand, the LEC $d_{1}^{V}$ multiplying the contact current is fitted to reproduce either $\sigma_{n p}$ in set II or $\mu_{V}$ in set III. Both alternatives still lead to somewhat large values for this LEC, but 
we find the degree of unnaturalness tolerable in this case. As we have remarked earlier, there are no three-body currents at N3LO, and therefore it is reasonable to fix the strength of this two-nucleon contact $M 1$ operator by fitting a three-nucleon observable such as $\mu_{V}$. Thus the set II (III) provides a prediction for $\mu_{V}\left(\sigma_{n p}\right)$. Cumulative contributions to these observables are shown in Fig. 4. These

$\left.\sigma_{\text {np }}^{\gamma} \quad \mu_{\mathrm{v}}{ }^{3} \mathrm{H} /{ }^{3} \mathrm{He}\right)$

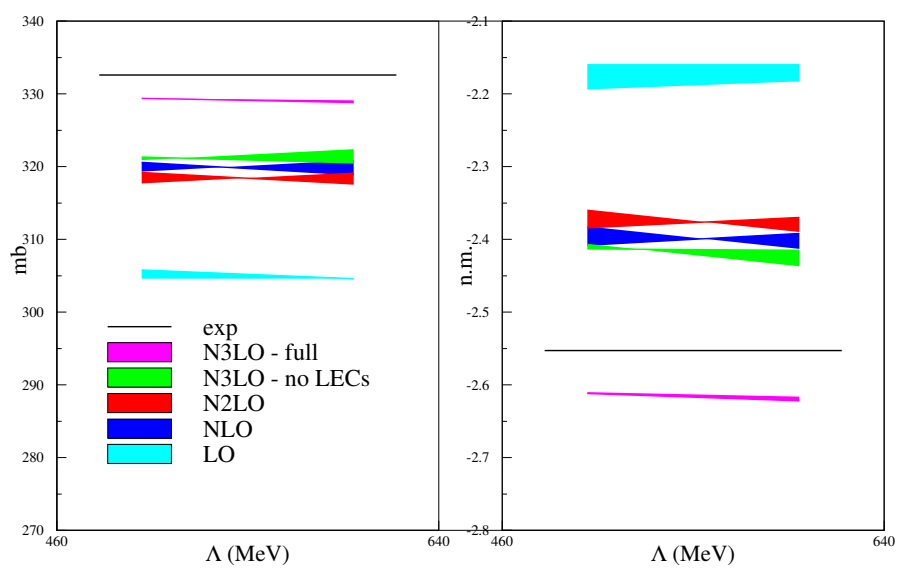

Figure 4: Cumulative contributions to the isovector trinucleon magnetic moment and to the $n p$ radiative capture cross sections. The black line indicates the experimental value. Notations as in Fig. 3.

predictions are within $2 \%$ for $\mu_{V}$ and $1 \%$ for $\sigma_{n p}$ of the experimental values, and exhibit a weak cutoff and Hamiltonian-model dependence.

\section{Conclusions}

Chiral perturbation theory, in conjunction with accurate ab-initio techniques to describe light nuclei, allows to make sharp predictions for nuclear electromagnetic observables in the low-energy domain. A recent investigation using quantum Montecarlo techniques, based on the model for the current described in this contribution, addressed the magnetic moments and transitions in nuclei with $A \leq 9$, reaching excellent agreement with experiments [24]. Static properties, including charge and magnetic radii and magnetic moments, and elastic form factors of $A=2,3$ nuclei were also investigated $[16,25,26]$.

We have described our formalism to derive the nuclear current and charge operators up to one loop order of the chiral expansion, based on the requirement that, when iterated in the LippmanSchwinger equation, they lead to the same transition amplitude obtained within time-ordered perturbation theory, order by order in the chiral expansion. In contrast to the authors of Ref. [10, 11], who use the unitary transformation method, we have not performed the complete renormalization of the one-pion exchange diagrams, but the momentum dependence of the corresponding magnetic moment operator turns out to be the same in the two formalisms. Further differences show up in the pion-loop corrections to the short range charge operators.

The issue of the convergence pattern of the chiral expansion and of the naturalness of the relevant LECs deserves further investigation, in particular for what concerns the role of the $\Delta$ resonance and its possible inclusion within the effective theory [27]. 
Acknowledgements The speaker would like to thank the organizers for a very stimulating Conference. The work of R.S. is supported by the U.S. Department of Energy, Office of Nuclear Science, under contract DEAC05-06OR23177.

\section{References}

[1] J. Gasser and H. Leutwyler, Annals Phys. 158, 142 (1984).

[2] V. Bernard, Prog. Part. Nucl. Phys. 60, 82 (2008).

[3] C. Ordonez, L. Ray and U. van Kolck, Phys. Rev. C 53, 2086 (1996).

[4] S. Weinberg, Phys. Lett. B251, 288 (1990); Nucl. Phys. B363, 3 (1991); Phys. Lett. B295, 114 (1992).

[5] S. Pastore, R. Schiavilla, and J.L. Goity, Phys. Rev. C 78, 064002 (2008).

[6] S. Pastore, L. Girlanda, R. Schiavilla, M. Viviani, and R.B. Wiringa, Phys. Rev. C 80, 034004 (2009).

[7] S. Pastore, L. Girlanda, R. Schiavilla, and M. Viviani, Phys. Rev. C 84, 024001 (2011).

[8] S. Okubo, Prog. Theor. Phys. 12, 603 (1954).

[9] E. Epelbaum, W. Glöckle, and U.-G. Meissner, Nucl. Phys. A637, 107 (1998); Nucl. Phys. A671, 295 (2000); Nucl. Phys. A714,535 (2003); Nucl. Phys. A747, 362 (2005).

[10] S. Kölling, E. Epelbaum, H. Krebs, and U.-G. Meissner, Phys. Rev. C80, 045502 (2009).

[11] S. Kölling, E. Epelbaum, H. Krebs, and U.-G. Meissner, Phys. Rev. C 84, 054008 (2011).

[12] T.-S. Park, D.-P. Min, and M. Rho, Nucl. Phys. A596, 515 (1996).

[13] J.L. Friar, Ann. Phys. (N.Y.) 104, 380 (1977).

[14] J.L. Friar, Phys. Rev. C 22, 796 (1980).

[15] J. Adam, H. Goller, and H. Arenhövel, Phys. Rev. C 48, 370 (1993).

[16] M. Piarulli, L. Girlanda, L. E. Marcucci, S. Pastore, R. Schiavilla, and M. Viviani, Phys. Rev. C 87 (2013) 014006.

[17] L. Girlanda, A. Kievsky, L.E. Marcucci, S. Pastore, R. Schiavilla, and M. Viviani, Phys. Rev. Lett. 105, 232502 (2010).

[18] A. Kievsky et al., J. Phys. G35, 063101 (2008).

[19] R.B. Wiringa, V.G.J. Stoks, and R. Schiavilla, Phys. Rev. C 51, 38 (1995).

[20] D.R. Entem and R. Machleidt, Phys. Rev. C 68, 041001 (2003).

[21] B.S. Pudliner et al., Phys. Rev. C 56, 1720 (1997).

[22] D. Gazit, S. Quaglioni, and P. Navratil, Phys. Rev. Lett. 103, 102502 (2009).

[23] R. Machleidt and D.R. Entem, Phys. Rep. 503, 1 (2011).

[24] S. Pastore, S. C. Pieper, R. Schiavilla, R. B. Wiringa and, arXiv:1212.3375 [nucl-th]. S. Pastore, these proceedings.

[25] M. Piarulli, poster at this Conference.

[26] S. Kolling, E. Epelbaum, and D. R. Phillips, Phys. Rev. C 86 (2012) 047001.

[27] H. Krebs, these proceedings. 